\title{
Juxtapapillary tumor as lung cancer's first manifestation
}

\begin{abstract}
Introduction: Metastatic carcinoma is the most prevalent intraocular neoplasm among adults. Usually, the ophthalmologist is the first physician detecting the spread of neoplasia, which in this case occurred as the first manifestation of lung cancer. Delayed diagnosis of this type of cancer leads to low rate survival over 5 years. Juxtapapillary ocular metastasis is a very rare lung cancer presentation, even rarer as the first manifestation of primary neoplasm. The aim of this report is to describe the occurrence of a juxtapapillary intraocular tumor as lung cancer's first sign.

Case report: Sixty-six years old female presents with complaints of visual impairment to an ophthalmologist, which detects intraocular tumor in the left eye. Retinography, fundus autofluorescence, fluorescein angiography, optical coherence tomography and ultras sonography were used to characterize tumor, which appeared in an unusual way: juxtapapillary. Clinical investigation was performed, the primary lesion was found in the lung and biopsy accused the adenocarcinoma type. Tumor regressed after chemotherapy, improving visual acuity.
\end{abstract}

Discussion: The intraocular tumor presentation was atypical and rare. About one third of patients have no primary cancer history at the intraocular metastasis diagnosis. The ophthalmologist should recognize intraocular tumors and refer the patient to the oncologist.
Volume 2 Issue 4 - 2015

\author{
Ricardo Luz Leit o Guerra,' Mylla Regina \\ Carneiro Santos, ${ }^{2}$ Ver nica Pontes Vilas Boas \\ Freitas,' Otac liode Oliveira Maia Junior, ${ }^{3}$ \\ Roberto Lorens Marback ${ }^{4}$ \\ 'Clinica de Olhos Leit o Guerra, Brazil \\ ${ }^{2}$ Escola Bahiana de Medicina e Sa de P blica, Brazil \\ ${ }^{3}$ Hospital S o Rafael, Brazil \\ ${ }^{4}$ Department of Ophthalmology, Faculdade de Medicina da Bahia \\ UFBA, Brazil
}

Correspondence: Ricardo Luz Leit o Guerra, Cl nica de Olhos Leit o Guerra Rua Catarina Paragua Gra a Salvador Bahia, Brazil,Tel +557। 35256555, Email ricardo@leitaoguerra.com.br

Received: April 25, 2015 | Published: May 06, 2015
Abbreviations: OD, right eye; OS, left eye; OU, both eyes; USG, ultrasonography; MRI, magnetic resonance imaging; OCT, optical coherence tomography; FAF, fundus autofluorescence; RPE, retinal pigment epithelium; FA, fluorescein angiography

\section{Introduction}

Lung carcinoma is one of the most incident cancers, and is the leading cause of cancer-related deaths in the world. In the United States, there are about 160.000 deaths $/$ year $^{1}$ and, in Brazil, approximately 22.424 deaths/year. ${ }^{2}$ Most patients have stage III or IV at diagnosis and rare lung carcinoma patients survive more than five years. ${ }^{3}$ Untreated patients in advanced stages and with metastatic involvement survive on average four months after diagnosis. ${ }^{4}$ There is a significant prevalence of ocular distant metastases in patients with extraocular malignancy. ${ }^{5}$ Metastatic carcinoma in the eye is the most prevalent intraocular cancer in adults. ${ }^{6}$ Usually, the ophthalmologist is the first physician detecting the spread of neoplasia, which is detected during eye examination, in patients with ocular symptoms such as decreased visual acuity and visual distortion. ${ }^{7}$ Metastasis of the optic disc can occur in a history of well-known cancer, but can sometimes be the first manifestation. ${ }^{8}$ Most of these tumors involve the choroid in an 8:1 ratio in relation to orbital metastases, ${ }^{6}$ but only $4.5 \%$ of all metastases affect intraocular optical disk with adjacent choroidal juxtapapillary involvement. Few descriptions found in the researched literature describes juxtapapillary tumor as yellowish lesions with diffuse thickening and flame shaped bleedings. ${ }^{8}$ The aim of this paper is to report a case of lung adenocarcinoma presenting ocular changes as the first clinical manifestation.

\section{Case report}

Sixty-six years old female presents complaining of decreased unilateral visual acuity on her left eye. Medical and family history had no relevant data. The ophthalmologic examination revealed visual acuity of 20/40 in the right eye (OD) and 20/100 in the left eye (OS). The slit lamp examination showed no change in both eyes $(\mathrm{OU})$ and the intraocular pressure was $14 \mathrm{mmHg}$ in OU. The fundus examination showed choroidal folds and drusen in the posterior pole of the OD (Figure 1A) and elevated hypo chromic lesion on the upper edge of the optic disc and choroid (Figure 1B). Complementary imaging examinations were performed: Fundus autofluorescence (FAF) (Figure 2A) (Figure 2B) fluorescein angiography (FA) (Figure 2C) (Figure 2D) and optical coherence tomography (OCT) (Figure 2E) (Figure 2F). Ultrasonography (USG) was performed on the left eye revealed intraocular tumor measuring $4.80 \mathrm{~mm} \times 12.93 \mathrm{~mm} \mathrm{x}$ $13.58 \mathrm{~mm}$ (HxLxT). The patient was referred for oncology evaluation. There were performed X-ray of the chest and full abdomen and a magnetic resonance imaging (MRI) of the chest. MRI revealed a tumor in the lower lobe of the right lung. Tumor excision followed by biopsy characterized as adenocarcinoma.

Chemotherapy was performed and the ophthalmologic evaluation 10 months after first ophthalmic examination showed similar findings in the OD. The OE showed visual acuity improvement to 20/60 and tumor disappearance with subretinal scar on site. (Figure 3) shows the pre and post treatment clinical aspect. The patient was advised to follow monitoring and possible treatment of the underlying disease with the oncologist. In a recent review, 20 months after diagnosis, the eye examination findings are stabilized. 




Figure I Fundus photography of both eyes IA) Right eye: Presence of choroidal folds anddrusen. IB) Left eye: Yellowish juxtapapillary tumor and adjacent choroidal folds.
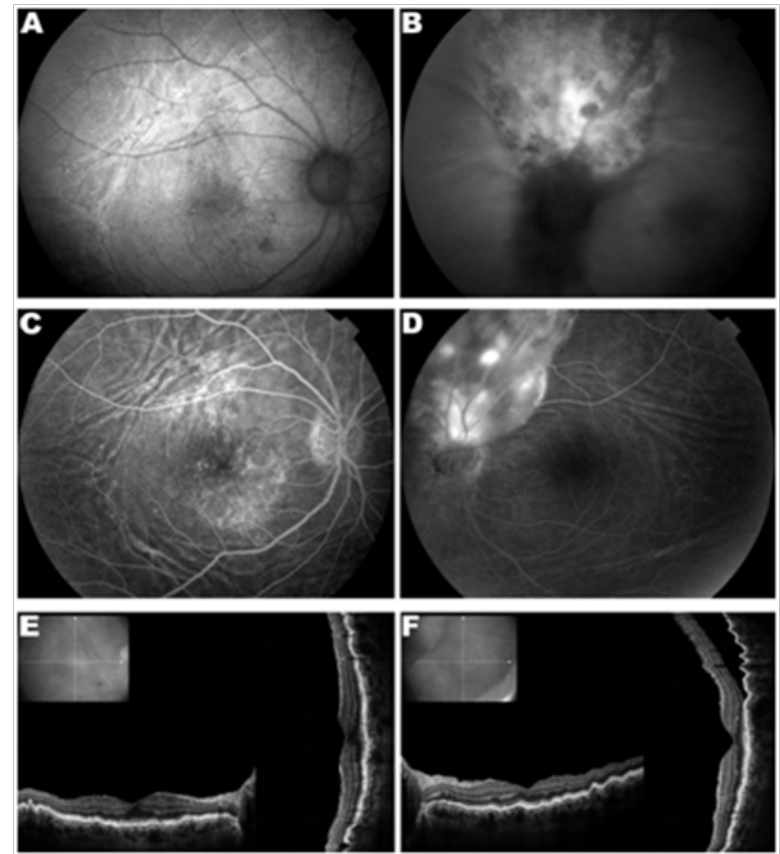

Figure 2 Complementary imaging examinations. (A) Fundus auto fluorescence (FAF) of the right eye (OD) highlighting choroidal folds and macular retinal pigment epithelium (RPE) changes. (B) FAF of the left eye (OS) presenting hiper and hipo autofluorescent areas in tumor surface. (C) OD fluorescein angiography (FAG) showing hyperfluorescence by window defect in the macular area and highlighting choroidal choroidal. (D) End stage of OS FAG presenting hiper and hipo fluoerescentare as at the tumor. (E) OD optical coherence tomography (OCT) showing in creasedtortuosity of the row for EPR dueto choroidal folds. (F) OS OCT shows serous retinal detachment superior and involving part of the macula. Choroidal folds can be seen especially in the upper and temporal areas.
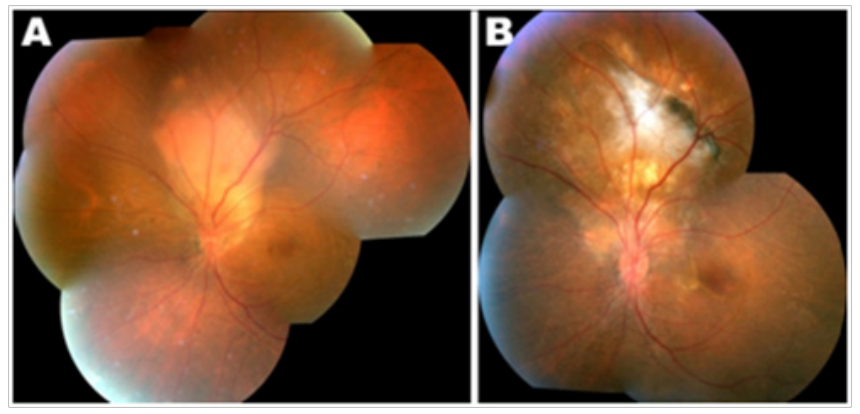

Figure 3 (A) Mosaic fundus photography at diagnosis. (B) Mosaic fundus photography after treatment.

\section{Discussion}

Historically it was known that ocular metastasis were rare. However, some studies show that its prevalence is higher than $20 \%$. The most common metastatic tumors are breast cancer, followed by lung cancer. ${ }^{9}$ About $80 \%$ of tumors have a unilateral effect, ${ }^{9}$ as well as the presented case. Choroid is the most affected intraocular structure $(61.9 \%)$ followed by retina $(28.5 \%)$, orbit $(4.8 \%)$ and optical disc (4.8). ${ }^{9}$ The presented patient had decreased best corrected visual acuity (20/100) in the affected eye, which is the main complaint of patients with metastatic tumors. ${ }^{9}$ Ocular metastasis affects mainly females. ${ }^{10}$ In a clinical study, patients with tumor with juxtapapillary components showed average of 13 months survival after ocular metastasis diagnosis. ${ }^{10}$ The presented patient follow-up remains stable for approximately 3 years since the first examination. Ocular Diagnosis is based on clinical findings in addition to imaging tests, such as: ocular USG, FAG, OCT. The differential diagnosis includes primary melanomas, benign lesions such as hemangioma and inflammatory granulomas. ${ }^{11}$ The diagnostic investigation of the referred case included the following complementary tests: FAF, FAG, OCT, ocular USG, X-ray, abdominal USG, MRI and lung biopsy through thoracotomy.

Studies justify the higher incidence of metastases in the left eye compared with the counter on the anatomical difference of the blood path, which is more tortuous to the right and therefore less susceptible to neoplastic cells. ${ }^{12}$ In the case presented, the tumor occurred in the left eye, and can be justified through this pathophysiological explanation. Treatment of intraocular tumors mainly includes radiotherapy and systemic chemotherapy ${ }^{13}$ followed by multidisciplinary team. The presented case showed anatomic and visual improvement after chemotherapy. About a third of patients have no primary cancer history at the time of ocular diagnosis. ${ }^{14}$ It is important that the ophthalmologists recognizes the tumor aspects and refer promptly to the oncologist.

\section{Acknowledgments}

None.

\section{Conflicts of interest}

Author declares that there is no conflict of interest.

\section{References}

1. National cancer institute. USA; 2015.

2. INCA. Instituto Nacional do Câncer. Número de mortes por câncer de pulmão. 2011.

3. Jamnik S, Santoro IL, Borges EL, et al. Comparative study of prognostic factors between two groups of patients with non-small cell lung cancer: survival above five years and below one year. Revista Brasileira de Cancerologia. 2009;55(1):5-10.

4. Novaes FT, Cataneo DC, Junior RLR, et al. Lung cancer: histology, staging, treatment and survival. J Bras Pneumol. 2008;34(8):595-600.

5. Junior EL, Herchenhorn D. Metástase intraocular, do diagnóstico à seleção terapêutica. Revista Onco \& janeiro/fevereiro; 2013.

6. Corrêa ZMS, Irion LCD, Marcon IM, et al. The diagnosis of metastatic carcinoma of the choroid using fine-needle aspiration biopsy (FNAB)Case report. Arq Bras Oftalmol. 2003;66:213-218.

7. Zhang HR, Ma ZZ, Feng Y, Guo T. Clinical characteristics of choroidal metastasis. Zhonghua Yan Ke Za Zhi. 2009; 45(4):301-308.

8. Banneux N, Bonnet S. Optic disc metastasis from pulmonary adenocarcinoma. Bull Soc Belge Ophtalmol. 2007;(305):79-84. 
9. Corrêa ZMS, Burmann TG, Freitas AM, et al. Prevalence of ocular metastasis in patients with known metastatic disease: preliminary results. Arq Bras Oftalmol. 2005;68(2):189-193.

10. Shields JA, Shields CL, Singh AD. Metastatic neoplasms in the optic disc: the 1999 Bjerrum Lecture: part 2. Arch Ophthalmol. 2000;118(2): 217224 .

11. Asteriou C, Konstantinou D, Kleontas A, et al. Blurred vision due to choroidal metastasis as the first manifestation of lung cancer: a case report. World J Surg Oncol. 2010;8:2.
12. Rivelli TG, Glasberg J, Vidoris A, et al. Metastatic breast carcinoma to the eye, eye lid and orbit: analysis of four cases treated in hospital. RBM Abr 12 V 69 Especial Oncologia 2.

13. Aragão REM, Barreira IMA, Gomes LMA, et al. Choroidal metastasis as the first sign of bronchioloalveolar lung cancer: case report. Arq Bras Oftalmol. 2013;76(4):250-252.

14. Shields CL, Shields JA, Gross NE, et al. Survey of 520 eyes with uveal metastases. Ophthalmology. 1997;104(8):1265-1276. 